\title{
Artificial boundary condition for a modified fractional diffusion problem
}

\author{
Abeeb A Awotunde ${ }^{1}$, Ryad A Ghanam² and Nasser-eddine Tatar ${ }^{3 *}$
}

\author{
"Correspondence: \\ tatarn@kfupm.edu.sa \\ ${ }^{3}$ Department of Mathematics and \\ Statistics, King Fahd University of \\ Petroleum and Minerals, Dhahran, \\ 31261, Saudi Arabia \\ Full list of author information is \\ available at the end of the article
}

\begin{abstract}
A diffusion problem involving a time derivative acting on two time scales represented by two fractional derivatives is investigated. The orders of the fractional derivatives are both between 0 and 1 and therefore the problem corresponds to the subdiffusion case. It is considered on a semi-infinite axis and the forcing term and the initial data are assumed compactly supported. To reduce the problem to that support there is a risk of being lead to an 'infected' problem due to the reflected waves on the new settled boundary. To avoid this undesirable effect of reflected waves on the standard boundaries, we establish artificial boundaries and find the appropriate artificial boundary conditions. Then, using the properties of fractional derivatives, a generalized version of the Mittag-Leffler function and some adequate manipulations of inverse Laplace transforms we find the explicit solution of the reduced problem.
\end{abstract}

\section{Introduction}

Fractional calculus is attracting a growing number of researchers these days due to its great capability of dealing with complex phenomena. In particular, fractional kinetic equations are shown to be more accurate in the treatment of anomalous diffusion. In petroleum engineering, the behavior of fluid flow in porous media is extremely difficult to describe. It has a non-Newtonian behavior which may be described much better using fractional derivatives.

In [1], Caputo, taking into account the memory effect, derived a new model by replacing the Darcy law [2] by a non-local one (in time) in a porous media problem (see also [3]). The equation looks like the usual diffusion equation but with a (Caputo type) fractional derivative placed in front of the Laplacian

$$
\frac{\partial p}{\partial t}=K D^{\alpha} \frac{\partial^{2} p}{\partial x^{2}} .
$$

This type of equations has been studied by few authors see [4-8], for instance, the sort of equation called the 'modified' fractional diffusion equation, whereas

$$
D^{\alpha} p=K \frac{\partial^{2} p}{\partial x^{2}}
$$

is called the fractional diffusion equation; see [9-12] where many interesting results have been derived. The latter equation is derived in detail in [7]. The normal (asymptotic long- 
time behavior of the) mean squared displacement $\left\langle r^{2}\right\rangle=M_{1} t$ is replaced by the 'generalized' mean squared displacement $\left\langle r^{2}\right\rangle=M_{\alpha} t^{\alpha}[13,14]$. The process is then categorized according to the values of $\alpha$ :

$$
\begin{aligned}
& 0<\alpha<1 \ldots \text { subdiffusive case, } \\
& \alpha=1 \ldots \text { ordinary diffusion equation, } \\
& 1<\alpha<2 \ldots \text { superdiffusive case, } \\
& \alpha=2 \ldots \text { wave equation. }
\end{aligned}
$$

A similar equation (to the one we intend to study),

$$
\frac{\partial p}{\partial t}=K_{1} D^{\alpha} \frac{\partial^{2} p}{\partial x^{2}}+K_{2} D^{\beta} \frac{\partial^{2} p}{\partial x^{2}},
$$

has been introduced and discussed in $[5,7,8,15-18]$ sometimes on the whole real axis. The second fractional derivative models the situation where the medium becomes less anomalous (see [10, 17-22]). It corresponds also to the mean squared displacement

$$
\left\langle r^{2}\right\rangle=M_{1} t^{1-\alpha}+M_{2} t^{1-\beta}
$$

justified by the observation that a single power law cannot describe accurately a complex anomalous phenomenon. This is the case for the oil flow in a fractal medium. Notice that when $0<\beta<\alpha<1$, at small times the first term in the right-hand side dominates whereas at large times it is the second term which dominates. For Riemann-Liouville derivatives, this behavior corresponds to accelerating diffusion and for Caputo derivatives it describes slowing-down subdiffusive processes.

It is worth noting that ultraslow diffusion is modeled by the fractional 'distributionalorder' equation $[5,7,23,24]$

$$
\int_{0}^{1} h(\gamma) D^{\gamma} p d \gamma=K \frac{\partial^{2} p}{\partial x^{2}}
$$

Accordingly, our two time scales equation becomes a special case corresponding to $h(\gamma)=$ $K_{1} \delta(\gamma-\alpha)+K_{2} \delta(\gamma-\beta)$ where $\delta$ is the Dirac distribution. We refer the reader to the review paper [25] (which complements and is a continuation of [20] by the same authors).

In petroleum engineering, oil is considered to be a viscoelastic material. As such it has the ability of storing energy and releasing it (dissipation) after removal of the load. The Boltzmann principle states that the relationship between the stress and the strain involves a time convolution of a kernel (called the relaxation function) and the Laplacian of the state

$$
\int_{0}^{t} \xi^{\prime}(t-s) \frac{\partial^{2} u}{\partial x^{2}}(s) d s
$$

The fractional derivative corresponds then to $\xi(t)=\frac{t^{-\alpha}}{\Gamma(1-\alpha)}$.

In general, the constitutive equation for the conventional viscoelastic models

$$
\sigma(t)+\sum_{k=1}^{m} a_{i} \frac{d^{k}}{d t^{k}} \sigma(t)=b_{0} \varepsilon(t)+\sum_{k=1}^{n} b_{i} \frac{d^{k}}{d t^{k}} \varepsilon(t)
$$


is replaced by (the fractional one) [26]

$$
\sigma(t)+\sum_{k=1}^{m} a_{i} D^{\gamma_{k}} \sigma(t)=b_{0} \varepsilon(t)+\sum_{k=1}^{n} b_{i} D^{\mu_{k}} \varepsilon(t), \quad 0<\gamma_{k}, \mu_{k}<1 .
$$

Moreover, it is well known in applications that lower order derivatives describe usually a certain type of damping (like the frictional damping represented by the first order derivative in hyperbolic problems). In the case of oil, it may be due to the different drag forces caused by the fractal media.

In this paper we would like to investigate the problem

$$
\left\{\begin{array}{l}
\frac{\partial p}{\partial t}=K_{1} D^{\alpha} \frac{\partial^{2} p}{\partial x^{2}}+K_{2} D^{\beta} \frac{\partial^{2} p}{\partial x^{2}}+f(x, t), \quad-L<x<\infty, t>0, \\
p(x, 0)=g(x), \quad-L<x<\infty \\
p(-L, t)=l(t), \quad t>0 \\
p(x, t) \rightarrow 0, \quad x \rightarrow+\infty, t>0 .
\end{array}\right.
$$

The function $p$ here is the pressure, $f$ and $g$ are compactly supported functions which denote an external source and the initial pressure at time $t=0$, respectively. The operators $D^{\alpha}$ and $D^{\beta}, 0<\beta<\alpha<1$ denote the Caputo fractional derivative of order $\alpha$ and $\beta$, respectively defined in the next section [27-31]; see also [29, 32, 33] for some interesting results.

Here, for continuous functions close to zero, the Caputo derivative and the RiemannLiouville derivative (see Definition 2 and Definition 3 below) are equivalent. The reason is that we shall be using the Laplace transform method and under this operator they have the same Laplace transform. These derivatives are equal for functions having the value zero at zero (see Theorem 1 below).

Initially and typically the problem is set in the semi-infinite axis $(0,+\infty)$ but for convenience we shall shift it to $(-L,+\infty)$. As the functions $f$ and $g$ are of compact support, we may, without loss of generality, assume that their support is in $(-L, 0)$. We first solve the problem in $(0,+\infty)$ (with $f$ and $g$ being zero). Then we solve the problem with all necessary (non-homogeneous) data in $(-L, 0)$. To be able to solve the problem in this bounded domain one needs to find appropriate boundary conditions which allow smooth movement of the flow and avoid the possible contamination of the solution. So these boundary conditions should not allow reflections which will contaminate the solution inside the bounded domain. Solving the problem first in $(0,+\infty)$ permits one to establish the appropriate (Neumann type) boundary condition at $x=0$ which is our artificial boundary. We mention here that artificial boundaries have been applied to other problems in [9, 34-39].

We shall use the Laplace transform and some crucial results due to Prabhakar (and others) as regards the three-parameter Mittag-Leffler function as well as some specific properties of fractional derivatives. In particular a crucial and problematic step of inverting Laplace transforms has been overcome by a simple trick.

The bounded domain is say $\left(L_{l}, L_{r}\right)$ but for simplicity we shall consider $(-L, 0)$ and the original unbounded domain is $(-L, \infty)$. This may be more convenient than considering $(0,1)$ and $(0, \infty)$. Our argument, once finished, will apply for the left semi-axis as well.

The plan of our paper is as follows: the next section contains some material needed in our argument later. In Section 3 we determine the appropriate artificial boundary condition. 
The proof of the uniqueness of the solution to the problem in the bounded domain can be found in Section 4. The explicit solution is derived in Section 5.

\section{Preliminaries}

We present in this section some material needed later to prove our result. They are collected from [27-31].

Definition 1 The Riemann-Liouville fractional integral of order $\alpha$ of $f$ is defined by

$$
\left(I_{0^{+}}^{\alpha} f\right)(t)=\frac{1}{\Gamma(\alpha)} \int_{0}^{t}(t-s)^{\alpha-1} f(s) d s, \quad t>0, \alpha>0,
$$

when the right-hand side exists.

Definition 2 The Riemann-Liouville fractional derivative of order $\alpha$ of $f$ is defined by

$$
\left({ }^{\mathrm{RL}} D_{0^{+}}^{\alpha} f\right)(t)=\frac{1}{\Gamma(1-\alpha)} \frac{d}{d t} \int_{0}^{t}(t-s)^{-\alpha} f(s) d s, \quad t>0,0<\alpha<1,
$$

when the right-hand side exists. Note that

$$
\left({ }^{\mathrm{RL}} D_{0^{+}}^{\alpha} f\right)(t)=\frac{d}{d t}\left(I^{1-\alpha} f\right)(t) .
$$

Definition 3 The Caputo fractional derivative of order $\alpha$ of $f$ is defined by

$$
\left({ }^{\mathrm{C}} D_{0^{+}}^{\alpha} f\right)(t)=\frac{1}{\Gamma(1-\alpha)} \int_{0}^{t}(t-s)^{-\alpha} f^{\prime}(s) d s, \quad t>0,0<\alpha<1
$$

(the prime here is for the derivative) when the right-hand side exists. Note that

$$
\left({ }^{\mathrm{C}} D_{0^{+}}^{\alpha} f\right)(t)=\left(I^{1-\alpha} \frac{d}{d t} f\right)(t) .
$$

The relationship between these two types of derivatives is given by the following theorem.

Theorem 1 We have

$$
\left({ }^{\mathrm{RL}} D_{0^{+}}^{\alpha} f\right)(t)=\left({ }^{\mathrm{C}} D_{0^{+}}^{\alpha} f\right)(t)+\frac{t^{-\alpha}}{\Gamma(1-\alpha)} f\left(0^{+}\right), \quad t>0,0<\alpha<1 .
$$

For $0<\alpha<1$, the Laplace transforms of these derivatives [40] are given by

$$
\begin{aligned}
& \mathcal{L}\left[\left(f^{\prime}\right)(t)\right](s)=s \mathcal{L}[f(t)](s)-f\left(0^{+}\right), \\
& \mathcal{L}\left[\left({ }^{C} D_{0^{+}}^{\alpha} f\right)(t)\right](s)=s^{\alpha} \mathcal{L}[f(t)](s)-s^{\alpha-1} f\left(0^{+}\right) .
\end{aligned}
$$

Lemma 2 Assume that $f(t)$ is continuous on $[0, A]$ for some $A>0$, then

$$
\lim _{t \rightarrow 0^{+}} I_{0^{+}}^{\alpha} f(t)=0, \quad \alpha>0 .
$$


Proof See [4].

Definition 4 A function $k \in L_{\text {loc }}^{1}[0,+\infty)$ is called positive definite if

$$
\int_{0}^{t} w(s) \int_{0}^{s} k(s-z) w(z) d z d s \geq 0, \quad t \geq 0,
$$

for every $w \in C[0,+\infty)$.

Definition 5 The function $k(t)$ is said to be strongly positive definite if there exists a positive constant $\gamma$ such that the mapping $t \rightarrow k(t)-\gamma e^{-t}$ is positive definite.

The function $k(t)=t^{-\alpha}, 0<\alpha<1$ is an example of a strongly positive definite function. In 1971 Prabhakar [41] proved the relation

$$
\mathcal{L}\left\{t^{\gamma-1} E_{\beta, \gamma}^{\delta}\left(w t^{\beta}\right)\right\}=s^{-\gamma}\left(1-w s^{-\beta}\right)^{-\delta}
$$

$\operatorname{Re} \beta>0, \operatorname{Re} \gamma>0, \operatorname{Re} s>0$, and $|s|>|w|^{\frac{1}{\operatorname{Re} \beta}}$, where $E_{\beta, \gamma}^{\delta}(z)$ is a generalized Mittag-Leffler function introduced by himself and defined by

$$
E_{\beta, \gamma}^{\delta}:=\sum_{n=0}^{\infty} \frac{(\delta)_{n} z^{n}}{\Gamma\left(\beta_{n}+\gamma\right) n !}, \quad \beta, \gamma, \delta \in \mathbb{C} \text { with } \operatorname{Re} \beta>0
$$

Here $(\delta)_{n}$ is the Pochhammer symbol

$$
(\delta)_{0}=1, \quad(\delta)_{n}=\delta(\delta+1)(\delta+2) \cdots(\delta+n-1) .
$$

The two-parameter Mittag-Leffler function corresponds to $\delta=1$ and the classical one corresponds to $\delta=\gamma=1$. We also recall the useful formula (see $[42,43])$

$$
\mathcal{L}^{-1}\left[\frac{s^{\alpha-\beta}}{s^{\alpha} \mp a}\right]=t^{\beta-1} E_{\alpha, \beta}\left( \pm a t^{\alpha}\right) .
$$

\section{Artificial boundary and artificial condition}

We designate

$$
\begin{aligned}
& \Omega=\{(x, t),-L \leq x<+\infty, 0<t \leq T\}, \\
& \Omega_{0}=\{(x, t),-L \leq x<0,0<t \leq T\}
\end{aligned}
$$

and

$$
\Omega_{e}=\{(x, t), 0 \leq x<+\infty, 0<t \leq T\} .
$$

Note that $\Omega$ is in this way split into a bounded domain $\Omega_{0}$ and an unbounded one $\Omega_{e}$. Our artificial boundary will be

$$
\Gamma_{0}=\{(x, t), x=0,0<t \leq T\} .
$$


Let us first determine the artificial boundary condition by solving the following problem:

$$
\left\{\begin{array}{l}
\frac{\partial p}{\partial t}=K_{1} D^{\alpha} \frac{\partial^{2} p}{\partial x^{2}}+K_{2} D^{\beta} \frac{\partial^{2} p}{\partial x^{2}} \quad \text { in } \Omega_{e} \\
p(x, 0)=0, \quad 0 \leq x<+\infty \\
p(0, t)=h(t), \quad 0<t<T, \\
p(x, t) \rightarrow 0 a s x \rightarrow+\infty, \quad 0<t \leq T
\end{array}\right.
$$

Here $0<\beta<\alpha<1$.

Applying the Laplace transform to both sides of the equation in (5) we find

$$
s \bar{p}(x, s)=K_{1} s^{\alpha} \frac{\partial^{2} \bar{p}}{\partial x^{2}}+K_{2} s^{\beta} \frac{\partial^{2} \bar{p}}{\partial x^{2}}=\left(K_{1} s^{\alpha}+K_{2} s^{\beta}\right) \frac{\partial^{2} \bar{p}}{\partial x^{2}} .
$$

The solution of this differential equation (in $x$ ) is equal to

$$
\bar{p}(x, s)=\bar{h}(s) e^{-x \sqrt{\frac{s}{K_{1} s^{\alpha}+K_{2} s^{\beta}}}}
$$

The inversion of this expression (7), however, is not easy. We are not aware of any (closed form) formula that may help. The ones that are available in the literature fall short either because of wrong signs in the powers or because of non-integer powers. In [15] the author has solved this problem by applying the Fourier transform. In our case this would not work. We will have to use either the Sine Fourier transform or the Cosine Fourier transform which unfortunately both will assume homogeneous Dirichlet boundary conditions or homogeneous Neumann conditions. We shall use the following trick (which will solve the difficulty circumvented and mentioned above regarding the wrong power signs):

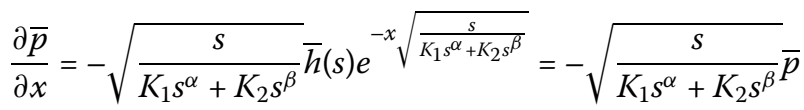

$$
\begin{aligned}
& =-\left(\frac{1}{s} \sqrt{\frac{s}{K_{1} s^{\alpha}+K_{2} s^{\beta}}}\right)(s \bar{p}) \text {. }
\end{aligned}
$$

Noticing that the inverse Laplace transform of $s \bar{p}$ is the time derivative of $p$, it remains only to invert the other expression. We have

$$
\frac{1}{s} \sqrt{\frac{s}{K_{1} s^{\alpha}+K_{2} s^{\beta}}}=\frac{s^{-1 / 2}}{\left(K_{1} s^{\alpha}+K_{2} s^{\beta}\right)^{1 / 2}} .
$$

Now that the power in $s^{-1 / 2}$ is negative; we can apply for instance the Prabhakar identity (3). Indeed,

$$
\frac{s^{-1 / 2}}{\left(K_{1} s^{\alpha}+K_{2} s^{\beta}\right)^{1 / 2}}=\frac{s^{-1 / 2}}{s^{\alpha / 2}\left(K_{1}+K_{2} s^{\beta-\alpha}\right)^{1 / 2}}=\frac{s^{-(\alpha+1) / 2}}{K_{1}^{1 / 2}\left(1+K_{2} K_{1}^{-1} s^{-(\alpha-\beta)}\right)^{1 / 2}},
$$

and its inverse Laplace transform is equal to

$$
\frac{1}{\sqrt{K_{1}}} t^{(\alpha-1) / 2} E_{\alpha-\beta,(\alpha+1) / 2}^{1 / 2}\left(-K_{2} K_{1}^{-1} t^{\alpha-\beta}\right) .
$$


Therefore

$$
\begin{aligned}
\frac{\partial p}{\partial x}(x, t) & =-\frac{1}{\sqrt{K_{1}}} t^{(\alpha-1) / 2} E_{\alpha-\beta,(\alpha+1) / 2}^{1 / 2}\left(-K_{2} K_{1}^{-1} t^{\alpha-\beta}\right) * \frac{\partial p}{\partial t} \\
& =-\frac{1}{\sqrt{K_{1}}} \int_{0}^{t} s^{(\alpha-1) / 2} E_{\alpha-\beta,(\alpha+1) / 2}^{1 / 2}\left(-K_{2} K_{1}^{-1} s^{\alpha-\beta}\right) \frac{\partial p}{\partial t}(x, t-s) d s
\end{aligned}
$$

and in the limit we find

$$
\begin{aligned}
\frac{\partial p}{\partial x}(0, t) & =-\frac{1}{\sqrt{K_{1}}} \int_{0}^{t} s^{(\alpha-1) / 2} E_{\alpha-\beta,(\alpha+1) / 2}^{1 / 2}\left(-K_{2} K_{1}^{-1} s^{\alpha-\beta}\right) \frac{\partial p}{\partial t}(0, t-s) d s \\
& =-\frac{1}{\sqrt{K_{1}}} \int_{0}^{t} s^{(\alpha-1) / 2} E_{\alpha-\beta,(\alpha+1) / 2}^{1 / 2}\left(-K_{2} K_{1}^{-1} s^{\alpha-\beta}\right) h^{\prime}(t-s) d s .
\end{aligned}
$$

As our equation reads $\frac{\partial p}{\partial t}=K_{1} D^{\alpha} \frac{\partial^{2} p}{\partial x^{2}}+K_{2} D^{\beta} \frac{\partial^{2} p}{\partial x^{2}}+f(x, t)$, it may be convenient to write the boundary condition as

$$
\begin{aligned}
& \left.\frac{\partial}{\partial x}\left(K_{1} D^{\alpha} p(x, t)+K_{2} D^{\beta} p(x, t)\right)\right|_{x=0} \\
& \quad=-\frac{1}{\sqrt{K_{1}}}\left(K_{1} D^{\alpha}+K_{2} D^{\beta}\right) \int_{0}^{t} s^{(\alpha-1) / 2} E_{\alpha-\beta,(\alpha+1) / 2}^{1 / 2}\left(-K_{2} K_{1}^{-1} s^{\alpha-\beta}\right) h^{\prime}(t-s) d s .
\end{aligned}
$$

Remark 1 Observe that if in (7) (instead of using the Prabhakar formula) we multiply and divide by $k$ ! and use the $k$ th derivative of the two-parameter Mittag-Leffler function $E_{\alpha, \beta}^{(k)}(t)$, then we obtain

$$
\mathcal{L}^{-1}\left\{\frac{k ! s^{(\beta-\alpha) k}}{\left(s^{1-\alpha}+K_{1} w^{2}\right)^{k+1}}\right\}=t^{k-\alpha-\beta k} E_{1-\alpha, 1-\alpha+k(\alpha-\beta)}^{(k)}\left(-K_{1} w^{2} t^{1-\alpha}\right) .
$$

To see the relationship of this derivative with the Prabhakar function we recall the formula (see [43])

$$
\left(\frac{d}{d z}\right)^{m} E_{\beta, \gamma}^{\delta}(z)=(\delta)_{m} E_{\beta, \gamma+m \beta}^{\delta+m}(z) .
$$

When $\delta=1$, we find

$$
E_{\beta, \gamma}^{(m)}(z)=(1)_{m} E_{\beta, \gamma+m \beta}^{1+m}(z)
$$

Taking $m=k$ and noting that $k !=(1)_{k}$ we may compare our result to the one in [16]. Moreover, recalling that (see [43])

$$
E_{\alpha, \beta}^{(\rho)}(z)=\frac{1}{\Gamma(\gamma)} H_{1,2}^{1,1}\left[-\left.z\right|_{(0,1),(1-\beta, \alpha)} ^{(1-\gamma, 1)}\right]
$$

where the right-hand side of (14) is the Fox $H$-function and

$$
\mathcal{M}\left[\mathcal{F}_{s}(f)\right][q](z)=\sqrt{\frac{2}{\pi}} \Gamma(z) \sin \frac{\pi z}{2} \mathcal{M}[f(x)](1-z),
$$


where $\mathcal{M}$ is for the Mellin transform, and proceeding as in [16], we may find an easier expression for (13). This expression is more suitable for numerical purposes.

The next lemma helps us differentiating the convolution term inside (13).

Lemma Assume that $f \in C^{1}([0, T])$, g continuous, and $0<\gamma<1$. Then

$$
\begin{aligned}
D^{\gamma}(f * g)(t) & =D^{\gamma}\left(\int_{0}^{t} f(t-\tau) g(\tau) d \tau\right) \\
& =f(0) I^{1-\gamma} g(t)+\int_{0}^{t} D^{\gamma} f(t-\tau) g(\tau) d \tau \\
& =f(0) I^{1-\gamma} g(t)+\left(D^{\gamma} f * g\right)(t), \quad t>0 .
\end{aligned}
$$

Proof We have from Definition 3

$$
\begin{aligned}
& D^{\gamma}\left(\int_{0}^{t} f(t-\tau) g(\tau) d \tau\right) \\
& \quad=I^{1-\gamma} D\left(\int_{0}^{t} f(t-\tau) g(\tau) d \tau\right) \\
& \quad=I^{1-\gamma}\left\{f(0) g(t)+\int_{0}^{t} f^{\prime}(t-\tau) g(\tau) d \tau\right\} \\
& \quad=f(0) I^{1-\gamma} g(t)+I^{1-\gamma}\left(\int_{0}^{t} f^{\prime}(t-\tau) g(\tau) d \tau\right) \\
& \quad=f(0) I^{1-\gamma} g(t)+\frac{1}{\Gamma(\gamma)} \int_{0}^{t}(t-s)^{\gamma-1} \int_{0}^{s} f^{\prime}(s-\tau) g(\tau) d \tau d s .
\end{aligned}
$$

By Fubini's theorem we have

$$
\begin{aligned}
& D^{\gamma}\left(\int_{0}^{t} f(t-\tau) g(\tau) d \tau\right) \\
& \quad=f(0) I^{1-\gamma} g(t)+\frac{1}{\Gamma(\gamma)} \int_{0}^{t}\left(\int_{\tau}^{t}(t-s)^{\gamma-1} f^{\prime}(s-\tau) d s\right) g(\tau) d \tau \\
& \quad=f(0) I^{1-\gamma} g(t)+\frac{1}{\Gamma(\gamma)} \int_{0}^{t}\left(\int_{\tau}^{t-\tau}(t-u-\tau)^{\gamma-1} f^{\prime}(u) d u\right) g(\tau) d \tau \\
& \quad=f(0) I^{1-\gamma} g(t)+\int_{0}^{t} D^{\gamma} f(t-\tau) g(\tau) d \tau .
\end{aligned}
$$

This completes the proof.

In our case $h(0)=0$ implies

$$
\begin{aligned}
\left(K_{1} D^{\alpha}\right. & \left.+K_{2} D^{\beta}\right) \int_{0}^{t} s^{(\alpha-1) / 2} E_{\alpha-\beta,(\alpha+1) / 2}^{1 / 2}\left(-K_{2} K_{1}^{-1} s^{\alpha-\beta}\right) h^{\prime}(t-s) d s \\
= & h(0)\left(K_{1} I^{1-\alpha}+K_{2} I^{1-\beta}\right) t^{(\alpha-1) / 2} E_{\alpha-\beta,(\alpha+1) / 2}^{1 / 2}\left(-K_{2} K_{1}^{-1} t^{\alpha-\beta}\right) \\
& \quad+\int_{0}^{t} s^{(\alpha-1) / 2} E_{\alpha-\beta,(\alpha+1) / 2}^{1 / 2}\left(-K_{2} K_{1}^{-1} s^{\alpha-\beta}\right)\left(K_{1} D^{\alpha}+K_{2} D^{\beta}\right) h^{\prime}(t-s) d s \\
= & \int_{0}^{t} s^{(\alpha-1) / 2} E_{\alpha-\beta,(\alpha+1) / 2}^{1 / 2}\left(-K_{2} K_{1}^{-1} s^{\alpha-\beta}\right)\left(K_{1} D^{\alpha}+K_{2} D^{\beta}\right) h^{\prime}(t-s) d s
\end{aligned}
$$


and consequently

$$
\begin{aligned}
& \left.\frac{\partial}{\partial x}\left(K_{1} D^{\alpha} p(x, t)+K_{2} D^{\alpha} p(x, t)\right)\right|_{x=0} \\
& \quad=-\frac{1}{\sqrt{K_{1}}} \int_{0}^{t} s^{(\alpha-1) / 2} E_{\alpha-\beta,(\alpha+1) / 2}^{1 / 2}\left(-K_{2} K_{1}^{-1} s^{\alpha-\beta}\right)\left(K_{1} D^{\alpha}+K_{2} D^{\beta}\right) h^{\prime}(t-s) d s .
\end{aligned}
$$

Remark 2 Note that, in the case the function $h(s)$ is not regular enough, then we can remedy it by multiplying and dividing by ' $K_{1} s^{\alpha}+K_{2} s^{\beta}$ ' instead of multiplying and dividing by ' $s$ ' in (8). The inverse Laplace transform of $\left(K_{1} s^{\alpha}+K_{2} s^{\beta}\right) \bar{p}$ is simply $\left(K_{1} D^{\alpha}+K_{2} D^{\beta}\right) p$.

Having found the artificial boundary condition (15) we may now consider the following equivalent problem on the bounded domain $\Omega_{0}$ :

$$
\left\{\begin{array}{l}
\frac{\partial p}{\partial t}=K_{1} D^{\alpha} \frac{\partial^{2} p}{\partial x^{2}}+K_{2} D^{\beta} \frac{\partial^{2} p}{\partial x^{2}}+f(x, t) \quad \text { in } \Omega_{0}, \\
p(x, 0)=g(x), \quad-L \leq x \leq 0, \\
p(-L, t)=l(t), \quad 0<t \leq T \\
\left.\frac{\partial}{\partial x}\left(K_{1} D^{\alpha} p(x, t)+K_{2} D^{\beta} p(x, t)\right)\right|_{x=0}=\Psi(t), \quad 0<t \leq T,
\end{array}\right.
$$

where $\Psi(t)$ is the right-hand expression in (15).

\section{Uniqueness of solution}

Theorem The solution of problem (16) is unique.

Proof Suppose, on the contrary, there exist two solutions $p_{1}$ and $p_{2}$, then $p_{0}=p_{1}-p_{2}$ is solution of the problem

$$
\left\{\begin{array}{l}
\frac{\partial p_{0}}{\partial t}=K_{1} D^{\alpha} \frac{\partial^{2} p_{0}}{\partial x^{2}}+K_{2} D^{\beta} \frac{\partial^{2} p_{0}}{\partial x^{2}} \quad \text { in } \Omega_{0}, \\
p_{0}(x, 0)=0, \quad-L \leq x \leq 0, \\
p_{0}(-L, t)=0, \quad 0<t \leq T, \\
\left.\frac{\partial}{\partial x}\left(K_{1} D^{\alpha} p_{0}(x, t)+K_{2} D^{\beta} p_{0}(x, t)\right)\right|_{x=0}=\Psi_{0}(t), \quad 0<t \leq T,
\end{array}\right.
$$

where

$$
\Psi_{0}(t)=\frac{-1}{\sqrt{K_{1}}} \int_{0}^{t} s^{(\alpha-1) / 2} E_{\alpha-\beta,(\alpha+1) / 2}^{1 / 2}\left(-K_{2} K_{1}^{-1} s^{\alpha-\beta}\right)\left(K_{1} D^{\alpha}+K_{2} D^{\beta}\right) p_{0}{ }^{\prime}(t-s) d s .
$$

Unlike the pure diffusion equation, here, because of the fractional derivative, the standard way of proving the uniqueness does not work. The main problem is a sign issue. Fortunately, the kernels in the definition of the fractional derivatives enjoy a nice property called 'positive definiteness' (see Definition 4). We shall make profit of this property. First, we multiply the equation in (17) by $\frac{\partial p_{0}}{\partial t}$ and integrate over $\Omega_{0}$, and we find

$$
\begin{aligned}
\int_{0}^{T} & \int_{-L}^{0}\left(\frac{\partial p_{0}}{\partial t}\right)^{2} d x d t \\
= & \int_{0}^{T} \int_{-L}^{0} K_{1} \frac{\partial p_{0}}{\partial t} D^{\alpha} \frac{\partial^{2} p_{0}}{\partial x^{2}} d x d t+\int_{0}^{T} \int_{-L}^{0} K_{2} \frac{\partial p_{0}}{\partial t} D^{\beta} \frac{\partial^{2} p_{0}}{\partial x^{2}} d x d t \\
= & \left.K_{1} \int_{0}^{T} \frac{\partial p_{0}}{\partial t} D^{\alpha} \frac{\partial p_{0}}{\partial x}\right|_{-L} ^{0} d t+\left.K_{2} \int_{0}^{T} \frac{\partial p_{0}}{\partial t} D^{\beta} \frac{\partial p_{0}}{\partial x}\right|_{-L} ^{0} d t \\
& -K_{1} \int_{0}^{T} \int_{-L}^{0} \frac{\partial^{2} p_{0}}{\partial x \partial t} D^{\alpha} \frac{\partial p_{0}}{\partial x} d x d t-K_{2} \int_{0}^{T} \int_{-L}^{0} \frac{\partial^{2} p}{\partial x \partial t} D^{\beta} \frac{\partial p_{0}}{\partial x} d x d t .
\end{aligned}
$$


From the definition of the Caputo fractional derivative (Definition 3) we have

$$
D^{\alpha} \frac{\partial p_{0}}{\partial x}(x, t)=\int_{0}^{t} \frac{(t-s)^{-\alpha}}{\Gamma(1-\alpha)} \frac{\partial^{2} p_{0}}{\partial t \partial x} d s
$$

and

$$
D^{\beta} \frac{\partial p_{0}}{\partial x}(x, t)=\int_{0}^{t} \frac{(t-s)^{-\beta}}{\Gamma(1-\beta)} \frac{\partial^{2} p_{0}}{\partial t \partial x} d s
$$

Therefore, the last two terms in the right-hand side of (19) are equal to

$$
-K_{1} \int_{0}^{T} \int_{-L}^{0} \frac{\partial^{2} p_{0}}{\partial x \partial t} \int_{0}^{t} \frac{(t-\tau)^{-\alpha}}{\Gamma(1-\alpha)} \frac{\partial^{2} p_{0}}{\partial t \partial x} d \tau d x d t \leq 0
$$

and

$$
-K_{2} \int_{0}^{T} \int_{-L}^{0} \frac{\partial^{2} p_{0}}{\partial x \partial t} \int_{0}^{t} \frac{(t-\tau)^{-\beta}}{\Gamma(1-\beta)} \frac{\partial^{2} p_{0}}{\partial t \partial x} d \tau d x d t \leq 0
$$

respectively. From (19) and the boundary conditions in (17) we deduce that

$$
\begin{aligned}
\int_{0}^{T} & \int_{-L}^{0}\left(\frac{\partial p_{0}}{\partial t}\right)^{2} d x d t+K_{1} \int_{0}^{T} \int_{-L}^{0} \frac{\partial^{2} p_{0}}{\partial x \partial t} \int_{0}^{t} \frac{(t-\tau)^{-\alpha}}{\Gamma(1-\alpha)} \frac{\partial^{2} p_{0}}{\partial t \partial x} d \tau d x d t \\
& +K_{2} \int_{0}^{T} \int_{-L}^{0} \frac{\partial^{2} p_{0}}{\partial x \partial t} \int_{0}^{t} \frac{(t-\tau)^{-\beta}}{\Gamma(1-\beta)} \frac{\partial^{2} p_{0}}{\partial t \partial x} d \tau d x d t \\
= & K_{1} \int_{0}^{T} \frac{\partial p_{0}}{\partial t}(0, t) D^{\alpha} \frac{\partial p_{0}}{\partial x}(0, t) d t+K_{2} \int_{0}^{T} \frac{\partial p_{0}}{\partial t}(0, t) D^{\beta} \frac{\partial p_{0}}{\partial t}(0, t) d t .
\end{aligned}
$$

The relations (19)-(21) show that the expression in the right-hand side of (22) is nonnegative. On the other hand, it is clear that the problem

$$
\left\{\begin{array}{l}
\frac{\partial q}{\partial t}=K_{1} D^{\alpha} \frac{\partial^{2} q}{\partial x^{2}}+K_{2} D^{\beta} \frac{\partial^{2} q}{\partial x^{2}} \quad \text { in } \Omega_{e}, \\
q(x, 0)=0, \quad 0<x<+\infty, \\
q(0, t)=p_{0}(0, t), \quad 0<t \leq T, \\
q(x, t) \rightarrow 0, \quad \text { as } x \rightarrow+\infty, 0<t \leq T,
\end{array}\right.
$$

possesses a unique solution. The multiplication of (23) by $\frac{\partial q}{\partial t}$ and the integration over $\Omega_{e}$ yield

$$
\begin{aligned}
\int_{0}^{T} & \int_{0}^{\infty}\left(\frac{\partial q}{\partial t}\right)^{2} d x d t \\
= & K_{1} \int_{0}^{T} \int_{0}^{\infty} \frac{\partial q}{\partial t} D^{\alpha} \frac{\partial^{2} q}{\partial x^{2}} d x d t+K_{2} \int_{0}^{T} \int_{-L}^{0} \frac{\partial q}{\partial t} D^{\beta} \frac{\partial^{2} q}{\partial x^{2}} d x d t \\
= & \left.K_{1} \int_{0}^{T} \frac{\partial q}{\partial t} D^{\alpha} \frac{\partial q}{\partial x}\right|_{0} ^{\infty} d t+\left.K_{2} \int_{0}^{T} \frac{\partial q}{\partial t} D^{\beta} \frac{\partial q}{\partial x}\right|_{0} ^{\infty} d t \\
& \quad-K_{1} \int_{0}^{T} \int_{0}^{\infty} \frac{\partial^{2} q}{\partial x \partial t} D^{\alpha} \frac{\partial q}{\partial x} d x d t-K_{2} \int_{0}^{T} \int_{0}^{\infty} \frac{\partial^{2} q}{\partial x \partial t} D^{\beta} \frac{\partial q}{\partial x} d x d t
\end{aligned}
$$


or

$$
\begin{aligned}
\int_{0}^{T} \int_{0}^{\infty}\left(\frac{\partial q}{\partial t}\right)^{2} d x d t \\
=-K_{1} \int_{0}^{T} \frac{\partial q}{\partial t}(0, t) D^{\alpha} \frac{\partial q}{\partial x}(0, t) d t-K_{2} \int_{0}^{T} \frac{\partial q}{\partial t}(0, t) D^{\beta} \frac{\partial q}{\partial x}(0, t) d t \\
\quad-K_{1} \int_{0}^{T} \int_{0}^{\infty} \frac{\partial^{2} q}{\partial x \partial t} \int_{0}^{t} \frac{(t-s)^{-\alpha}}{\Gamma(1-\alpha)} \frac{\partial^{2} q}{\partial t \partial x} d s d x d t \\
\quad-K_{2} \int_{0}^{T} \int_{0}^{\infty} \frac{\partial^{2} q}{\partial x \partial t} \int_{0}^{t} \frac{(t-s)^{-\beta}}{\Gamma(1-\beta)} \frac{\partial^{2} q}{\partial t \partial x} d s d x d t
\end{aligned}
$$

Noting that $\frac{\partial q}{\partial t}(0, t)=\frac{\partial p_{0}}{\partial t}(0, t)$ and $\left.\frac{\partial q}{\partial x}\right|_{x=0}=\left.\frac{\partial p_{0}}{\partial t}\right|_{x=0}$ we conclude from $(24)$ and the positive definiteness of the kernels that the expression

$$
-K_{1} \int_{0}^{T} \frac{\partial p_{0}}{\partial t}(0, t) D^{\alpha} \frac{\partial p_{0}}{\partial x}(0, t) d t-K_{2} \int_{0}^{T} \frac{\partial p_{0}}{\partial t}(0, t) D^{\beta} \frac{\partial p_{0}}{\partial x}(0, t) d t
$$

i.e. the sum of the first two terms in the right-hand side of (24), is nonnegative. This fact, together with the one of the first part of the proof, implies that this expression is equal to zero. It follows from $(22)$ that $\frac{\partial p_{0}}{\partial t}(x, t)=0$ for all $0 \leq x<\infty$ and $0 \leq t \leq T$. As $p_{0}(-L, T)=0$ we conclude that $p_{0} \equiv 0$ and thus the solution of (16) is unique.

\section{Explicit solution of (16) in $\boldsymbol{\Omega}_{\mathbf{0}}$}

Let us look for a solution to (16) in the form of the sum of a transient solution and a steadystate solution,

$$
p(x, t)=U(x, t)+V(x, t) .
$$

By substitution into (16) we find

$$
\frac{\partial U}{\partial t}+\frac{\partial V}{\partial t}=K_{1} D^{\alpha}\left(\frac{\partial^{2} U}{\partial x^{2}}+\frac{\partial^{2} V}{\partial x^{2}}\right)+K_{2} D^{\beta}\left(\frac{\partial^{2} U}{\partial x^{2}}+\frac{\partial^{2} V}{\partial x^{2}}\right)+f(x, t)
$$

and the boundary conditions give

$$
\begin{aligned}
& U(-L, t)+V(-L, t)=l(t), \\
& \left.\frac{\partial}{\partial x}\left[K_{1} D^{\alpha}(U(x, t)+V(x, t))+K_{2} D^{\beta}(U(x, t)+V(x, t))\right]\right|_{x=0}=\Psi(t) .
\end{aligned}
$$

The problem will be partitioned into

$$
\left\{\begin{array}{l}
\frac{\partial^{2} V}{\partial x^{2}}=0 \quad \text { in } \Omega_{0}, \\
V(-L, t)=l(t), \quad 0<t \leq T, \\
\left.\frac{\partial}{\partial x}\left[K_{1} D^{\alpha} V(x, t)+K_{2} D^{\beta} V(x, t)\right]\right|_{x=0}=\Psi(t), \quad 0<t \leq T
\end{array}\right.
$$

and

$$
\left\{\begin{array}{l}
\frac{\partial U}{\partial t}=K_{1} D^{\alpha} \frac{\partial^{2} U}{\partial x^{2}}+K_{2} D^{\beta} \frac{\partial^{2} U}{\partial x^{2}}-\frac{\partial V}{\partial t}+f(x, t) \quad \text { in } \Omega_{0} \\
U(-L, t)=0, \quad 0<t \leq T \\
\left.\frac{\partial}{\partial x}\left[K_{1} D^{\alpha} U(x, t)+K_{2} D^{\beta} U(x, t)\right]\right|_{x=0}=0, \quad 0<t \leq T \\
U(x, 0)=g(x)-V(x, 0), \quad-L \leq x \leq 0 .
\end{array}\right.
$$


We start by solving problem (26). We may look for a solution in the form

$$
V(x, t)=v_{1}(t) x+v_{2}(t)
$$

The boundary conditions imply

$$
\begin{aligned}
& v_{2}(t)-L v_{1}(t)=l(t), \\
& K_{1} D^{\alpha} v_{1}(t)+K_{2} D^{\beta} v_{1}(t)=\Psi(t) .
\end{aligned}
$$

Therefore, from (28) and (29) we see that

$$
V(x, t)=(L+x) v_{1}(t)+l(t) .
$$

The function $v_{1}(t)$ may be found by the Laplace transform method. Indeed, assuming continuity of $v_{1}(t)$ at zero, we have

$$
K_{1} s^{\alpha} \bar{v}_{1}(s)+K_{2} s^{\beta} \bar{v}_{1}(s)=\bar{\Psi}(s)
$$

or

$$
\bar{v}_{1}(s)=\frac{\bar{\Psi}(s)}{K_{1} s^{\alpha}+K_{2} s^{\beta}}=\frac{\bar{\Psi}(s)}{K_{1}} \frac{s^{-\beta}}{s^{\alpha-\beta}+\frac{K_{2}}{K_{1}}} .
$$

Using (4), i.e.

$$
\mathcal{L}^{-1}\left[\frac{s^{\alpha-\beta}}{s^{\alpha} \mp a}\right]=t^{\beta-1} E_{\alpha, \beta}\left( \pm a t^{\alpha}\right),
$$

we see that

$$
\mathcal{L}^{-1}\left[\frac{s^{-\beta}}{s^{\alpha-\beta}+K_{2} K_{1}^{-1}}\right]=t^{\alpha-1} E_{\alpha-\beta, \alpha}\left(-\frac{K_{2}}{K_{1}} t^{\alpha-\beta}\right) .
$$

Therefore

$$
v_{1}(t)=\left(\Psi * t^{\alpha-1} E_{\alpha-\beta, \alpha}\left(-\frac{K_{2}}{K_{1}} t^{\alpha-\beta}\right)\right)(t) .
$$

We pass now to problem (27). We write the equation there as

$$
\frac{\partial U}{\partial t}=K_{1} D^{\alpha} \frac{\partial^{2} U}{\partial x^{2}}+K_{2} D^{\beta} \frac{\partial^{2} U}{\partial x^{2}}+\tilde{f}(x, t)
$$

with $\tilde{f}(x, t)=f(x, t)-\frac{\partial V}{\partial t}$. We look for a solution in the form

$$
U(x, t)=\sum_{n=0}^{\infty} X_{n}(x) T_{n}(t)
$$


We assume that

$$
\tilde{f}(x, t)=\sum_{n=0}^{\infty} A_{n}(t) X_{n}(x),
$$

where the coefficients $A_{n}(t)$ may be computed by

$$
A_{n}(t)=\left(\int_{-L}^{0} \tilde{f}(x, t) X_{n}(x) d x\right)\left(\int_{-L}^{0} X_{n}(x) X_{n}(x) d x\right)^{-1} .
$$

The substitution of the expressions (34) and (35) in (33) yields

$$
\begin{aligned}
\frac{\partial}{\partial t}\left(\sum_{n=0}^{\infty} T_{n}(t) X_{n}(x)\right)= & K_{1} D^{\alpha}\left(\sum_{n=0}^{\infty} T_{n}(t) X^{\prime \prime}{ }_{n}(x)\right) \\
& +K_{2} D^{\beta}\left(\sum_{n=0}^{\infty} T_{n}(t) X^{\prime \prime}{ }_{n}(x)\right)+\sum_{n=0}^{\infty} A_{n}(t) X_{n}(x) .
\end{aligned}
$$

Before continuing with this identity we need to solve the homogeneous problem

$$
\left\{\begin{array}{l}
\frac{\partial W}{\partial t}=K_{1} D^{\alpha} \frac{\partial^{2} W}{\partial x^{2}}+K_{2} D^{\beta} \frac{\partial^{2} W}{\partial x^{2}} \quad \text { in } \Omega_{e} \\
W(-L, t)=0, \quad 0<t \leq T \\
\left.\frac{\partial}{\partial x}\left[K_{1} D^{\alpha} W(x, t)+K_{2} D^{\beta} W(x, t)\right]\right|_{x=0}=0, \quad 0<t \leq T .
\end{array}\right.
$$

Setting $W(x, t)=X(x) T(t)$, we obtain by differentiation and substitution in (37)

$$
\begin{aligned}
X(x) T^{\prime}(t) & =K_{1} X^{\prime \prime}(x) T^{(\alpha)}(t)+K_{2} X^{\prime \prime}(x) T^{(\beta)}(t) \\
& =\left[K_{1} T^{(\alpha)}(t)+K_{2} T^{(\beta)}(t)\right] X^{\prime \prime}(x)
\end{aligned}
$$

and separating variables it appears that

$$
\frac{T^{\prime}(t)}{K_{1} T^{(\alpha)}(t)+K_{2} T^{(\beta)}(t)}=\frac{X^{\prime \prime}(x)}{X(x)}=\mu=-\lambda^{2} .
$$

The first boundary condition $W(-L, t)=0$ implies $X(-L) T(t)=0,0<t \leq T$ and consequently $X(-L)=0$. The second boundary condition gives

$$
K_{1} X^{\prime}(0) T^{(\alpha)}(t)+K_{2} X^{\prime}(0) T^{(\beta)}(t)=0, \quad 0<t \leq T
$$

or

$$
\left[K_{1} T^{(\alpha)}(t)+K_{2} T^{(\beta)}(t)\right] X^{\prime}(0)=0, \quad 0<t \leq T .
$$

We conclude that $X^{\prime}(0)=0$. Solving the problem

$$
\left\{\begin{array}{l}
X^{\prime \prime}(x)+\lambda^{2} X(x)=0,-\quad L \leq x \leq 0, \\
X(-L)=0, \quad X^{\prime}(0)=0
\end{array}\right.
$$

we obtain $X_{n}(x)=\cos \lambda_{n} x$ with $\lambda_{n}=\frac{(2 n+1) \pi}{2 L}$. These eigenfunctions form a basis. 
Now, in view of (36) we deduce that

$$
\sum_{n=0}^{\infty} T^{\prime}{ }_{n}(t) X_{n}(x)=\sum_{n=0}^{\infty}\left[K_{1} T^{(\alpha)}(t)+K_{2} T^{(\beta)}(t)\right]\left(-\lambda_{n}^{2} X_{n}(x)\right)+\sum_{n=0}^{\infty} A_{n}(t) X_{n}(x) .
$$

We get the system of equations

$$
T_{n}^{\prime}(t)+\lambda_{n}^{2}\left[K_{1} T_{n}^{(\alpha)}(t)+K_{2} T_{n}^{(\beta)}(t)\right]=A_{n}(t), \quad 0<t \leq T .
$$

Notice that

$$
U(x, 0)=\sum_{n=0}^{\infty} X_{n}(x) T_{n}(0)=g(x)-V(x, 0)=g(x)-l(0)=g(x)-g(-L) .
$$

This means that $T_{n}(0)$ are the coefficients of the expansion of $g(x)-g(-L)$ according to the basis $\left\{X_{n}(x)\right\}_{n}$.

Applying Laplace transform to (38) we end up with

$$
s \bar{T}_{n}(s)+\lambda_{n}^{2}\left[K_{1} s^{\alpha} \bar{T}_{n}(s)+K_{2} s^{\beta} \bar{T}_{n}(s)\right]=\bar{A}_{n}(s)+T_{n}(0)
$$

or

$$
\left[s+\lambda_{n}^{2}\left(K_{1} s^{\alpha}+K_{2} s^{\beta}\right)\right] \bar{T}_{n}(s)=\bar{A}_{n}(s)+T_{n}(0) .
$$

That is,

$$
\bar{T}_{n}(s)=\frac{\bar{A}_{n}(s)+T_{n}(0)}{s+\lambda_{n}^{2}\left(K_{1} s^{\alpha}+K_{2} s^{\beta}\right)} .
$$

Note that

$$
\begin{aligned}
& \frac{1}{s+} \lambda_{n}^{2}\left(K_{1} s^{\alpha}+K_{2} s^{\beta}\right) \\
& =\frac{s^{-\beta}}{s^{1-\beta}+K_{1} \lambda_{n}^{2} s^{\alpha-\beta}+K_{2} \lambda_{n}^{2}}=\frac{s^{-\beta}}{\left(s^{1-\beta}+K_{2} \lambda_{n}^{2}\right)\left(1+\frac{K_{1} \lambda_{n}^{2} s^{\alpha-\beta}}{s^{1-\beta}+K_{2} \lambda_{n}^{2}}\right)} \\
& =\frac{s^{-\beta}}{s^{1-\beta}+K_{2} \lambda_{n}^{2}} \sum_{k=0}^{\infty}\left(\frac{-K_{1} \lambda_{n}^{2} s^{\alpha-\beta}}{s^{1-\beta}+K_{2} \lambda_{n}^{2}}\right)^{k}=\sum_{k=0}^{\infty} \frac{\left(-K_{1} \lambda_{n}^{2}\right)^{k} s^{-\beta+k(\alpha-\beta)}}{\left(s^{1-\beta}+K_{2} \lambda_{n}^{2}\right)^{k+1}}
\end{aligned}
$$

Using (3) we see that

$$
\mathcal{L}^{-1}\left\{\frac{s^{-\beta+k(\alpha-\beta)}}{\left(s^{1-\beta}+K_{2} \lambda_{n}^{2}\right)^{k+1}}\right\}=t^{k(1-\alpha)} E_{1-\beta, k(1-\alpha)+1}^{k+1}\left(-K_{2} \lambda_{n}^{2} t^{1-\beta}\right) .
$$

Therefore (39)-(41) imply that

$$
\begin{aligned}
T_{n}(t)= & \int_{0}^{t} A_{n}(t-s) \sum_{k=0}^{\infty}\left(-K_{1} \lambda_{n}^{2}\right)^{k} s^{k(1-\alpha)} E_{1-\beta, k(1-\alpha)+1}^{k+1}\left(-K_{2} \lambda_{n}^{2} s^{1-\beta}\right) d s \\
& +T_{n}(0) \sum_{k=0}^{\infty}\left(-K_{1} \lambda_{n}^{2}\right)^{k} t^{k(1-\alpha)} E_{1-\beta, k(1-\alpha)+1}^{k+1}\left(-K_{2} \lambda_{n}^{2} t^{1-\beta}\right)
\end{aligned}
$$


and by (34) we have

$$
U(x, t)=\sum_{n=0}^{\infty} T_{n}(t) \cos \frac{(2 n+1) \pi}{2 L} x
$$

with $T_{n}(t)$ as in (42). Gathering (43), (42), (32), and (31) in (25) we obtain the solution $p(x, t)$ to the problem in $\Omega_{0}$.

As a conclusion we have proved the following result.

Theorem 3 The solution of problem (16) is given by (25) where $U(x, t)$ is as in (43). The functions $v_{1}(t)$ in $V(x, t)($ see (31)) is given in (32). That is,

$$
\begin{aligned}
p(x, t)= & \sum_{n=0}^{\infty} \cos \frac{(2 n+1) \pi}{2 L} x \\
& \times\left[\int_{0}^{t} A_{n}(t-s) \sum_{k=0}^{\infty}\left(-K_{1} \lambda_{n}^{2}\right)^{k} s^{k(1-\alpha)} E_{1-\beta, k(1-\alpha)+1}^{k+1}\left(-K_{2} \lambda_{n}^{2} s^{1-\beta}\right) d s\right. \\
& \left.+T_{n}(0) \sum_{k=0}^{\infty}\left(-K_{1} \lambda_{n}^{2}\right)^{k} t^{k(1-\alpha)} E_{1-\beta, k(1-\alpha)+1}^{k+1}\left(-K_{2} \lambda_{n}^{2} t^{1-\beta}\right)\right] \\
& +(L+x)\left(\Psi * t^{\alpha-1} E_{\alpha-\beta, \alpha}\left(-\frac{K_{2}}{K_{1}} t^{\alpha-\beta}\right)\right)(t)+l(t),
\end{aligned}
$$

where

$$
\Psi(t)=\frac{-1}{\sqrt{K_{1}}} \int_{0}^{t} s^{(\alpha-1) / 2} E_{\alpha-\beta,(\alpha+1) / 2}^{1 / 2}\left(-K_{2} K_{1}^{-1} s^{\alpha-\beta}\right)\left(K_{1} D^{\alpha}+K_{2} D^{\beta}\right) h^{\prime}(t-s) d s,
$$

$A_{n}(t)$ and $T_{n}(0)$ are the coefficients of the expansions of $\tilde{f}(x, t)=f(x, t)-\frac{\partial V}{\partial t}$ and $g(x)-g(-L)$, respectively, according to the basis $\left\{X_{n}(x)\right\}_{n}$.

The graphs (see Figure 1) correspond to $p(x, 0)=g(x)=\sin (4 \pi x), p(-1, t)=l(t)=h(t)=t$.

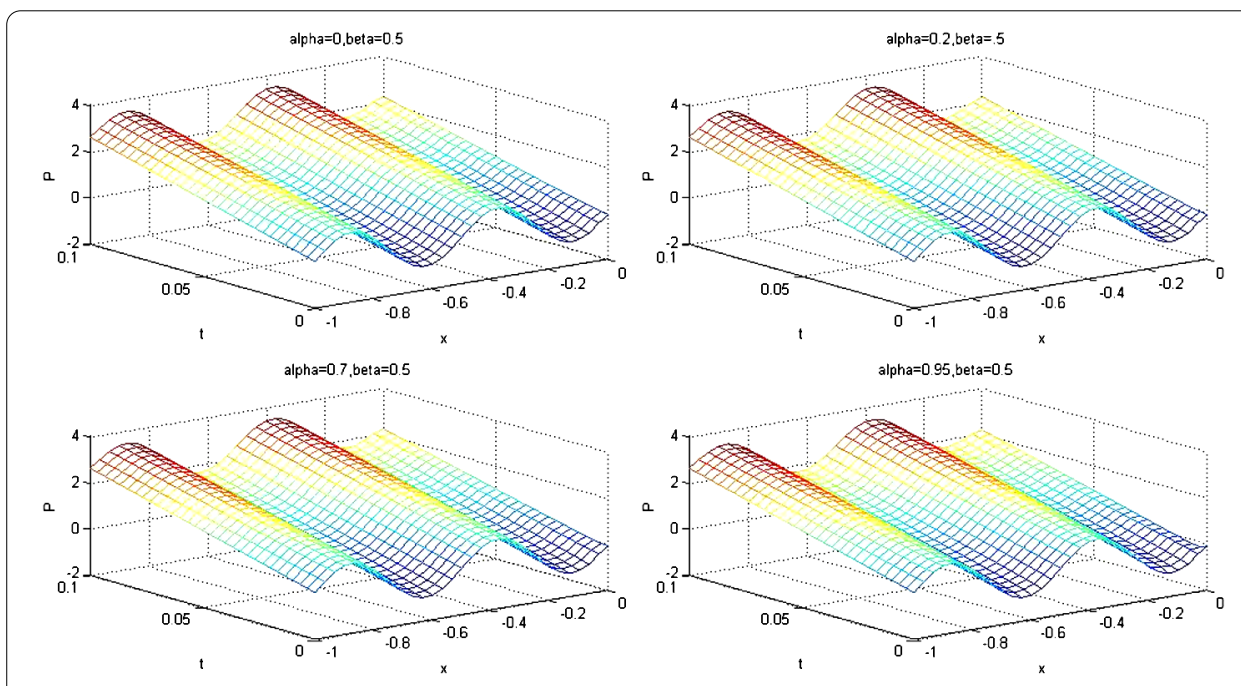

Figure 1 The graphs of $\boldsymbol{p}(\boldsymbol{x}, \boldsymbol{t}) . p(x, 0)=g(x)=\sin (4 \pi x) ; p(-l, t)=I(t)=h(t)=t$. 


\section{Competing interests}

The authors declare that they have no competing interests.

\section{Authors' contributions}

The authors have contributed almost equally. Dr. R Ghanam and Dr. A Awotunde have produced the graphs. All authors read and approved the final manuscript.

\section{Author details}

${ }^{1}$ Petroleum Engineering Department, King Fahd University of Petroleum and Minerals, Dhahran, 31261, Saudi Arabia. ${ }^{2}$ Department of Mathematics, Virginia Commonwealth University in Qatar, Doha, Qatar. ${ }^{3}$ Department of Mathematics and Statistics, King Fahd University of Petroleum and Minerals, Dhahran, 31261, Saudi Arabia.

\section{Acknowledgements}

The authors would like to acknowledge the support provided by King Abdulaziz City for Science and Technology (KACST) through the Science and Technology Unit at King Fahd University of Petroleum and Minerals (KFUPM) for funding this work through project No. 11-OIL1663-04 as part of the National Science, Technology and Innovation Plan.

Received: 28 June 2014 Accepted: 2 January 2015 Published online: 31 January 2015

\section{References}

1. Caputo, M: Diffusion of fluids in porous media with memory. Geothermics 28, 113-130 (1999)

2. Darcy, H: Les Fontaines Publiques de la Ville de Dijon. Dalmont, Paris (1856)

3. Malik, NA, Ghanam, RA, Al-Homidan, S: Sensitivity of the pressure distribution to the fractional order $\alpha$ in the fractional diffusion equation. Can. J. Phys. 93, 1-19 (2015). doi:10.1139/cjp-2013-0387

4. Al-Homidan, S, Ghanam, RA, Tatar, N-e: On a generalized diffusion equation arising in petroleum engineering. Adv. Differ. Equ. 2013, 349 (2013)

5. Chechkin, AV, Gorenflo, R, Sokolov, IM, Gonchar, VY: Distributed order time fractional diffusion equation. Fract. Calc. Appl. Anal. 6(3), 259-279 (2003)

6. Shen, F, Tan, WC, Zhao, Y, Masuoka, T: The Rayleigh-Stokes problem for a heated generalized second grade fluid with fractional derivative model. Nonlinear Anal., Real World Appl. 7(5), 1072-1080 (2006)

7. Sokolov, IM, Chechkin, AV, Klafter, J: Distributed-order fractional kinetics. Acta Phys. Pol. B 35(4), 1323-1341 (2004)

8. Sokolov, IM, Klafter, J: From diffusion to anomalous diffusion: a century after Einstein's Brownian motion. Chaos, Interdiscip. J. Nonlinear Sci. 15, 026103 (2005). doi:10.1063/1.1860472

9. Han, H, Zhu, L, Brunner, H, Ma, J: The numerical solution of parabilic Volterra integro-differential equations on unbounded spatial domains. Appl. Numer. Math. 55, 83-99 (2005)

10. LV, LJ, Xiao, JB, Zhang, L, Gao, L: Solutions for a generalized fractional anomalous diffusion equation. J. Comput. Appl. Math. 225(1), 301-308 (2009)

11. Salim, TO, El-Kahlout, A: Solution of fractional order Rayleigh-Stokes equations. Adv. Theor. Appl. Mech. 1(5), 241-254 (2008)

12. Sandev, T, Metzler, R, Tomovski, Z: Fractional diffusion equation with a generalized Riemann-Liouville time fractional derivative. J. Phys. A, Math. Theor. 44, 255203 (2011)

13. Saxton, MJ: Anomalous diffusion due to obstacles: a Monte Carlo study. Biophys. J. 66, 394-401 (1994)

14. Saxton, M: Anomalous subdiffusion in fluorescence photobleaching recovery: a Monte Carlo study. Biophys. J. 81 , 2226-2240 (2001)

15. Langlands, TAM: Solution of a modified fractional diffusion equation. Physica A 367, 136-144 (2006)

16. Langlands, TAM, Henry, BI, Wearne, SN: Fractional cable equation models for anomalous electrodiffusion in nerve cells: infinite domain solutions. J. Math. Biol. 59(6), 761-808 (2009)

17. Langlands, TAM, Henry, BI, Wearne, SN: Fractional cable equation models for anomalous electrodiffusion in nerve cells: finite domain solutions. SIAM J. Appl. Math. 71(4), 1168-1203 (2011)

18. Liu, F, Yang, C, Burrage, K: Numerical method analytical technique of the modified anomalous subdiffusion equation with a nonlinear source term. J. Comput. Appl. Math. 231(1), 160-176 (2009)

19. Chen, CM, Liu, F, Anh, V: A Fourier method and an extrapolation technique for Stokes' first problem for a heated generalized second grade fluid with fractional derivative. J. Comput. Appl. Math. 223, 777-789 (2009)

20. Metzler, R, Klafter, J: The random walk's guide to anomalous diffusion: a fractional dynamics approach. Phys. Rep. 339 1-77 (2000)

21. Tan, WC, Pan, WX, Xu, MY: A note on unsteady flows of a viscoelastic fluid with the fractional Maxwell model between two parallel plates. Int. J. Non-Linear Mech. 38, 645-650 (2003)

22. Tan, WC, Xu, MY: Unsteady flows of a generalized second grade fluid with the fractional derivative model between two parallel plates. Acta Mech. Sin. 20, 471-476 (2004)

23. Atanackovic, TM, Pilipovic, S, Zorica, D: Existence and calculation of the solution to the time distributed order diffusion equation. Phys. Scr. T 136, 014012 (2009). doi:10.1088/0031-8949/2009/T136/014012

24. Saberi Najafi, H, Refahi Sheikhani, A, Ansari, A: Stability analysis of distributed order fractional differential equations. Abstr. Appl. Anal. 2011, Article ID 175323 (2011). doi:10.1155/2011/175323

25. Metzler, R, Klafter, J: The restaurant at the end of the random walk: recent developments in the description of anomalous transport by fractional dynamics. J. Phys. A, Math. Gen. 37, R161 (2004). doi:10.1088/0305-4470/37/31/R01

26. Pritz, T: Five-parameter fractional derivative model for polymeric damping materials. J. Sound Vib. 265(5), 935-952 (2003)

27. Kilbas, AA, Srivastava, HM, Trujillo, JJ: Theory and Applications of Fractional Differential Equations. North-Holland Mathematics Studies (van Mill, J (ed.)), vol. 204. Elsevier, Amsterdam (2006)

28. Miller, KS, Ross, B: An Introduction to the Fractional Calculus and Fractional Differential Equations. Wiley, New York (1993)

29. Nyamoradi, N, Baleanu, D, Agarwal, RP: Existence and uniqueness of positive solutions to fractional boundary value problems with nonlinear boundary conditions. Adv. Differ. Equ. 2013, 266 (2013) 
30. Podlubny, I: Fractional Differential Equations. Mathematics in Sciences and Engineering, vol. 198. Academic Press, San Diego (1999)

31. Samko, SG, Kilbas, AA, Marichev, Ol: Fractional Integrals and Derivatives: Theory and Applications. Gordon \& Breach, Yverdon (1993)

32. Darzi, R, Mohammadzadeh, B, Neamaty, A, Baleanu, D: Lower and upper solutions method for positive solutions of fractional boundary value problems. Abstr. Appl. Anal. 2013, Article ID 847184 (2013). doi:10.1155/2013/847184

33. Zhang, L, Baleanu, D, Wang, G: Nonlocal boundary value problem for nonlinear impulsive $q(k)$-integrodifference equation. Abstr. Appl. Anal. 2014, Article ID 478185 (2014)

34. Gao, GH, Sun, ZZ, Zhang, YN: A finite difference scheme for fractional sub-diffusion equations on an unbounded domain using artificial boundary conditions. J. Comput. Phys. 231, 2865-2879 (2012)

35. Ghanam, RA, Malik, N, Tatar, N-e: Transparent boundary conditions for a diffusion problem modified by Hilfer derivative. J. Math. Sci. Univ. Tokyo 21, 129-152 (2014)

36. Han, H, Huang, Z: A class of artificial boundary conditions for heat equation in unbounded domain. Comput. Math. Appl. 43, 889-900 (2002)

37. Han, H, Zhu, L, Brunner, H, Ma, J: Artificial boundary conditions for parabolic Volterra integro-differential equations on unbounded two-dimensional domains. J. Comput. Appl. Math. 197, 406-420 (2006)

38. Wu, X, Sun, ZZ: Convergence of difference scheme for heat equation in unbounded domains using artificial boundary conditions. Appl. Numer. Math. 50, 261-277 (2004)

39. Wu, X, Zhang, J: High-order local absorbing boundary conditions for heat equation in unbounded domains. J. Comput. Math. 29(1), 74-90 (2011)

40. Kexue, L, Jigen, P: Laplace transform and fractional differential equations. Appl. Math. Lett. 24, 2019-2023 (2011)

41. Prabhakar, TR: A singular integral equation with a generalized Mittag-Leffler function in the kernel. Yokohama Math. J. $19,7-15(1971)$

42. Haubold, HJ, Mathai, AM, Saxena, RK: Mittag-Leffler functions and application. J. Appl. Math. 2011, Article ID 298628 (2011)

43. Rubens, FC, Capelas de Olivera, E, Vaz, J Jr:: On the generalized Mittag-Leffler function and its application in a fractional telegraph equation. Math. Phys. Anal. Geom. 15, 1-16 (2012)

\section{Submit your manuscript to a SpringerOpen ${ }^{\circ}$ journal and benefit from:}

- Convenient online submission

- Rigorous peer review

- Immediate publication on acceptance

- Open access: articles freely available online

- High visibility within the field

- Retaining the copyright to your article 